\title{
CONSTRUCTION OF MAGNETIC DICHROISM BEAM LINE IN TRIESTE
}

\author{
F. LAMA \\ Istituto di Struttura della Materia/CNR, via E. Fermi 38, 00044 Frascati, Italy
}

The described synchrotron radiation beam line; based on a double monochromator with different incidence angles, has been devised to ally in a short length the working possibilities of both vacuum ultraviolet and soft X-ray monochromators as well as to preserve the degree of circular polarization of the radiation emitted by the source. The design philosophy and the predicted performances of the diffraction and the focusing systems are described and the heat load on the beam line optical elements is discussed. Some practical details concerning the beam line, now under construction, are presented.

PACS numbers: $42.15 . \mathrm{Eq}, 07.85 . \mathrm{Qe}, 42.79 . \mathrm{Yd}$

\section{Introduction}

Circularly polarized synchrotron radiation (CPR) in the vacuum ultraviolet and soft X-ray regions has very recently become one of the most important achievement for the researchers involved in the study of magnetism. Not only the well-stated absorption or reflection measurement [1-3] techniques are able to give magnetic information on elements and sites, involving transitions from core levels, but element specific magnetic hysteresis [4] and circular polarized magnetic scattering [5] have also been demonstrated to be powerful new techniques for studying the magnetic properties of matter.

Nowadays, CPR is mainly used while studying magnetic properties of ultra-thin films and multilayers to develop new materials with greatly magnetic storage capacity but it is also an ideal tool while investigating materials that exhibit dichroism or other different absorption characteristic with respect to right or left handed circularly polarized radiation.

Moreover even for non magnetic and non chiral systems CPR is of fundamental importance in studying, for instance, the spin polarization characteristic of electrons emitted following the photoionization of atoms, molecules and solids.

As in the case of the majority of third generation synchrotron radiation sources also in Trieste, at ELETTRA, a project to obtain circularly polarized radiation from an insertion device is under development. The CPR will be emitted from an electromagnetic elliptical wiggler (EEW) presently on its final definition [6]. 
The EEW forces the electrons to orbit on an helical path using two perpendicular arrays of magnets and will be able to deliver elliptically polarized radiation from $5 \mathrm{eV}$ to $1200 \mathrm{eV}$. In the horizontal direction the magnetic field will be produced by using an array of electromagnets while in the vertical direction the intensity of the vertical magnetic field varies by changing the width of the gap between the two arrays of permanent magnets.

The choice of the magnetic parameters makes the EEW suitable for operating in undulator as well as in wiggler mode allowing to optimize its performances in the entire photon energy range.

The electrons helical path and consequently the circular polarization of the emitted radiation could be changed from left handed to right handed with a switching rate of about $100 \mathrm{~Hz}$, by applying an alternate current to the electromagnets.

\section{Design consideration}

The will to maintain the degree of circular polarization of radiation as well as the total photon flux on the entire energy range delivered by the EEW convinced us to design a beam line equipped with two interchangeable monochromators sharing the same entrance and exit slits, a normal incidence monochromator for the lower part of the photon energy range and a grazing incidence one for the higher part.

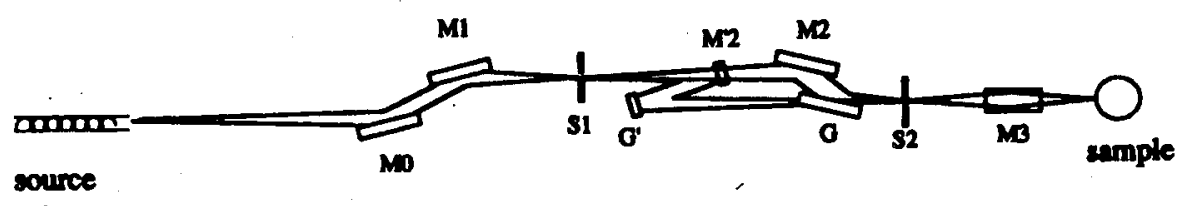

Fig. 1. General layout of the beam line.

Figure 1 shows the side view of the beam line. A solid angle of $\left(0.9_{\text {vert }} \times\right.$ $\left.2_{\text {horiz }}\right) \operatorname{mrad}^{2}$ of the radiation emitted by the EEW is collected and vertically diverted $(5 \mathrm{deg})$ by the first plane mirror $\left(M_{0}\right)$ of the beam line. Then the toroidal mirror $\left(M_{1}\right)$ focuses the radiation vertically (grazing angle of $2.5 \mathrm{deg}$ ) onto the entrance slit $\left(S_{1}\right)$ and horizontally onto the exit slit $\left(S_{2}\right)$ with magnification 0.3 and 1.8 , respectively.

After the entrance slit the radiation could be diverted, towards the two interchangeable, normal incidence, holographically corrected, spherical gratings $\left(G_{1,2}^{\prime}\right)$, inserting along the optical path a cylindrical $\left(M_{2}^{\prime}\right)$ convex mirror with an incidence angle of $1.22 \mathrm{deg}$. The $M_{2}^{\prime}$ mirror restores a stigmatic source onto the entrance slit for the gratings $\left(G_{1,2}^{\prime}\right)$. The radiation diffracted by the gratings is then stigmatically focused onto the exit slit $S_{2}$. This normal incidence monochromators (NIM) covers the photon energy range from $5 \mathrm{eV}$ to $35 \mathrm{eV}$.

Moving apart the mirror $M_{2}^{\prime}$ allows the radiation to hit a grazing incidence plane mirror $\left(M_{2}\right)$ that deflects the radiation towards one out of four interchangeable grazing incidence lamellar concave gratings $\left(G_{1-4}\right)$. Following a scheme proposed by H.A. Padmore [7] the combined rotation of the $M_{2}$ and $G_{1-4}$ is able to maintain onto the exit slit a fixed focusing condition while scanning the diffracted 
wavelengths. This spherical grazing incidence monochromators (SGM) is dedicated to the photon energy region from $30 \mathrm{eV}$ to $1200 \mathrm{eV}$.

Finally, after the exit slit, a toroidal mirror $\left(M_{3}\right)$ at grazing incidence with an unitary magnification transfers the exit slit image onto the sample inside the experimental chamber.

One of the most challenging problem met during the project work was the evaluation of the mirror surface figure errors induced by the absorption of the soft $\mathrm{X}$-ray emitted from the EEW. We performed numerically evaluation of the degree of deformation of all the heated surfaces for every working condition of the EEW with respect to different blank materials and water cooling systems.

Nowadays, silicon carbide, silicon and Glidcop are widely used in manufacturing synchrotron radiation beam line optical elements. An analysis using finite element calculations of three-dimensional thermal stress allows us to choose silicon as the most suitable material for the $M_{0}$ mirror blank and to estimate the expected

TABLE

The most important parameters for the optical elements of the circular polarization beam line.

\begin{tabular}{|c|c|c|c|c|c|c|c|}
\hline $\begin{array}{l}\text { Mirr. } \\
\& \\
\text { Grat. }\end{array}$ & $\begin{array}{c}\text { Shape\& } \\
\text { Incidence } \\
\text { angle (deg) }\end{array}$ & $\begin{array}{l}R(\mathrm{~m}) \\
\text { tang. }\end{array}$ & $\begin{array}{l}\rho(\mathrm{m}) \\
\text { sag. }\end{array}$ & $\begin{array}{l}\text { Illuminated } \\
\text { area }\left[\mathrm{mm}^{2}\right]\end{array}$ & $\begin{array}{c}\text { Slope err. } \\
\text { tang. \& } \\
\text { sag. } \\
\text { (RMS) }\end{array}$ & $\begin{array}{c}\text { Power } \\
\text { load \& } \\
\text { Water } \\
\text { cooling }\end{array}$ & $\begin{array}{c}\text { Blank } \\
\text { materials } \\
\text { \&coatings }\end{array}$ \\
\hline$M_{0}$ & $\begin{array}{c}\text { Flat } \\
\theta=87.5\end{array}$ & $\infty$ & $\infty$ & $360 \times 35$ & $<2^{\prime \prime}, 2^{\prime \prime}$ & $\begin{array}{c}<265 \mathrm{~W} \\
\text { lateral }\end{array}$ & $\begin{array}{c}\text { Silicon } \\
\mathrm{Au}\end{array}$ \\
\hline$\overline{M_{1}}$ & $\begin{array}{l}\text { Toroidal } \\
\theta=87.5\end{array}$ & 229.254 & 0.629 & $410 \times 40$ & $<0.5^{\prime \prime}, 2^{\prime \prime}$ & $\begin{array}{c}<37.2 \mathrm{~W} \\
\text { lateral }\end{array}$ & $\begin{array}{c}\text { Silicon } \\
\mathrm{Au}\end{array}$ \\
\hline$M_{2}$ & $\begin{array}{c}\text { Flat } \\
\theta>82\end{array}$ & $\infty$ & $\infty$ & $400 \times 27$ & $<0.5^{\prime \prime}, 0.5^{\prime \prime}$ & $\begin{array}{c}<29.8 \mathrm{~W} \\
\text { lateral }\end{array}$ & $\begin{array}{c}\text { CVD SiC } \\
\mathrm{Au}\end{array}$ \\
\hline$M_{2}^{\prime}$ & $\begin{array}{c}\text { Cylindrical } \\
\theta=1.22\end{array}$ & $\infty$ & -2.309 & $10 \times 10$ & $<2^{\prime \prime}, 2^{\prime \prime}$ & $\begin{array}{c}<39.1 \mathrm{~W} \\
\text { lateral }\end{array}$ & $\begin{array}{c}\text { CVD SiC } \\
\mathrm{Au}\end{array}$ \\
\hline$\overline{G_{1}}$ & $\begin{array}{c}\text { Spherical } \\
\theta>87.5\end{array}$ & 42.830 & 42.830 & $180 \times 27$ & $<0.5^{\prime \prime}, 0.5^{\prime \prime}$ & $\begin{array}{c}<11.0 \mathrm{~W} \\
\text { lateral }\end{array}$ & $\begin{array}{c}\text { CVD SiC } \\
\mathrm{Au}\end{array}$ \\
\hline$\overline{G_{2}}$ & $\begin{array}{c}\text { Spherical } \\
\theta>86.8\end{array}$ & 33.70 & 33.70 & $180 \times 27$ & $<0.5^{\prime \prime}, 0.5^{\prime \prime}$ & $\begin{array}{c}<11.6 \mathrm{~W} \\
\text { lateral }\end{array}$ & $\begin{array}{c}\text { CVD SiC } \\
\text { Au }\end{array}$ \\
\hline$\overline{G_{3}}$ & $\begin{array}{c}\text { Spherical } \\
\theta>84\end{array}$ & 22.945 & 22.945 & $107 \times 27$ & $<0.5^{\prime \prime}, 0.5^{\prime \prime}$ & $\begin{array}{c}<12.0 \mathrm{~W} \\
\text { lateral }\end{array}$ & $\begin{array}{c}\text { CVD SiC } \\
\mathrm{Au}\end{array}$ \\
\hline$G_{4}$ & $\begin{array}{c}\text { Sperical } \\
\theta>82\end{array}$ & 17.129 & 17.129 & $87 \times 27$ & $<0.5^{\prime \prime}, 0.5^{\prime \prime}$ & $\begin{array}{c}<12.3 \mathrm{~W} \\
\text { lateral }\end{array}$ & $\begin{array}{c}\text { CVD SiC } \\
\mathrm{Au}\end{array}$ \\
\hline$\overline{G_{1}^{\prime}}$ & $\begin{array}{l}\text { Sperical } \\
\theta<1.22\end{array}$ & 3.002 & 3.002 & $10 \times 10$ & $<0.5^{\prime \prime}, 0.5^{\prime \prime}$ & $\begin{array}{c}<0.20 \mathrm{~W} \\
\text { absent }\end{array}$ & $\begin{array}{c}\text { Fused } \\
\text { Silica } \\
\mathrm{Al}+\mathrm{MgF}_{2}\end{array}$ \\
\hline$G_{2}^{\prime}$ & $\begin{array}{l}\text { Sperical } \\
\theta<1.22\end{array}$ & 3.002 & 3.002 & $10 \times 10$ & $<0.5^{\prime \prime}, 0.5^{\prime \prime}$ & $\begin{array}{c}<0.20 \mathrm{~W} \\
\text { absent }\end{array}$ & $\begin{array}{c}\text { Fused } \\
\text { Silica } \\
\mathrm{Au}\end{array}$ \\
\hline$M_{3}$ & $\begin{array}{l}\text { Toroidal } \\
\theta=87.4\end{array}$ & 45.790 & 0.087 & $160 \times 20$ & $<1^{\prime \prime}, 2^{\prime \prime}$ & $\begin{array}{c}<0.58 \mathrm{~W} \\
\text { absent }\end{array}$ & $\begin{array}{c}\text { Zerodur } \\
\mathrm{Au}\end{array}$ \\
\hline
\end{tabular}


degradation of its optical performances. Moreover, these calculations suggest a lateral water cooling and a flat shape for the first mirror of the beam line and allow us to define better cooling solutions for all other optical elements. The value of the expected thermal induced maximum slope error on the $M_{0}$ surface is smaller than 2 arcsec.

In Table we summarize the main parameters for the beam line optical elements.

\section{Expected performances}

In the final version of the design of the beam line consistent effort was dedicated in order to obtain the best compromise between the needs of high resolution and that of high flux of circularly polarized photons.

For the NIM monochromator the contribution of optical aberrations to the resolution limit is mainly due to the defocusing term. Using an ion etched holographically corrected (type IV) concave grating limit the defocusing aberration contribution allows one to maintain the position of the grating fixed while it is rotated to scan the different wavelengths. Figure 2 shows the root mean square (RMS) linear dispersion obtainable using the NIM equipped with two type IV gratings with a complementary optimized working range.

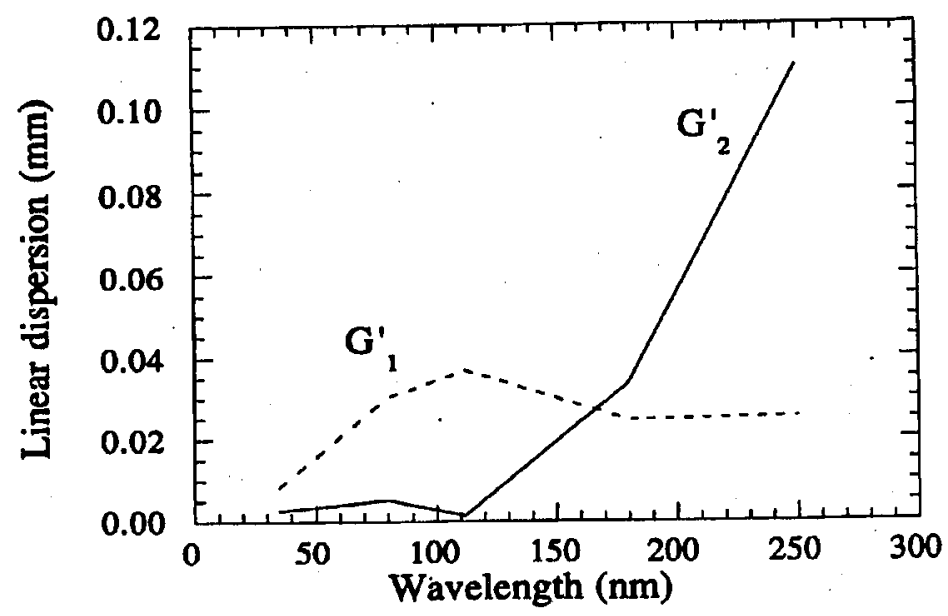

Fig. 2. RMS lineár dispersion calculated onto the exit slit position vs. photons wavelengths for the normal incidence gratings.

In the case of the SGM monochromator the reduction of the defocusing contribution is obtained through the variation of the gratings included angle as a function of the photon energy. The variation of the included angle is obtained while rotating the premirror $M_{2}$. In Fig. 3 the resolving power obtainable in this configuration is reported. The coma aberration contribution is responsible for the degradation of the resolution performances in the high part of the energy range of each spectrum. This contribution could be limited reducing the dimension of the acceptance angle of the gratings. 


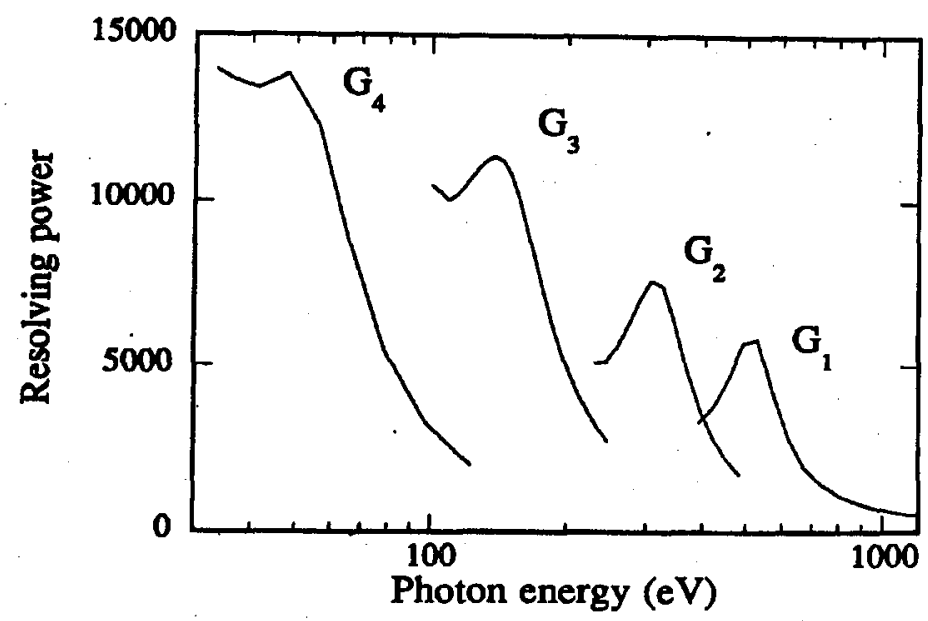

Fig. 3. Resolving power calculated considering the vectorial sum of the first order optical aberrations, slope errors, entrance and exit slits contributions for the grazing incidence gratings.

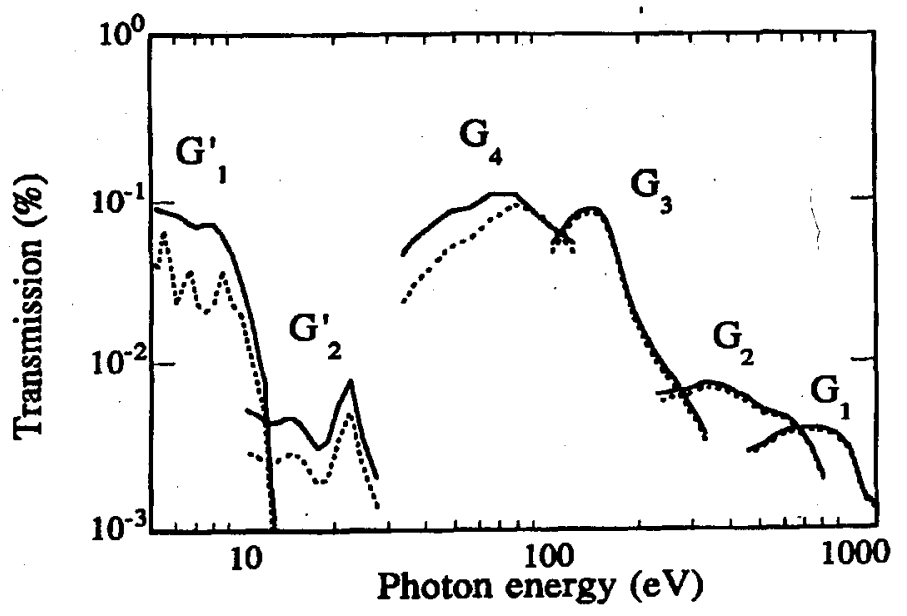

Fig. 4. Percentage of the total transmitted photon flux available at the sample position for the $s$ (solid line) and $p$ (dashed line) polarization components of the radiation emitted from the EEW.

The choices of the grating characteristics as well as of their working angles represent the best compromise to exploit the properties of the radiation emitted from the EEW. Considering the reflecting coatings of the optical elements, as reported in Table, we performed a computer simulation to calculate the total transmission of the entire beam line. The non optimized grating efficiencies were calculated using the differential equation formalism described by Neviere et al. [8]. The final results are shown in Fig. 4. 
Considering an average degree of circular polarization of $80 \%$ and an average total photon flux of $1 \times 10^{14}$ (photons/s/ $/ 0.01 \%$ band width) on the entire photon energy range of interest, the worst degree of circular polarization available at the sample position will be of $70 \%$ with a total flux of $1 \times 10^{12}$ (photons/s/0.01\%band width).

\section{Recent achievements}

Mechanical tolerances and stability of the beam line components are of great importance in achieving the expected performances. For this reason we spent a lot of attention in the design, realization and test of the mechanics and apparatus. The use of mirror manipulators allow them to rotate, around their pole, along the direction of the radiation and perpendicular to it, for the mirrors $M_{0}, M_{1}$ and $M_{3}$ with a precision of $\pm 2.5 \mu \mathrm{rad}$. The translations $(x, y, z)$ and the remaining rotation are performed by means of precision (up to $\pm 5 \mu \mathrm{m}$ and $\pm 5 \mu \mathrm{rad}$, respectively) screws, positioned outside the vacuum chambers. The alignment of the optical elements inside the two monochromators $\left(M_{2}^{\prime}, G_{1,2}^{\prime}\right.$ and $\left.M_{2}, G_{1-4}\right)$ has made possible to move each optical element holder with a precision up to $\pm 2.0 \mu \mathrm{rad}$ while rotating and up to $\pm 5.0 \mu \mathrm{m}$ while translating. The scanning rotations are obtained by means of long levers capable to maintain a linear response along the entire angle range with a precision of $\pm 2.0 \mu \mathrm{rad}$.

Precision, tolerance and stability performances of every mechanical part was tested and the results are almost equal or even better with respect to the design specifications.

Each one of the vacuum chambers and their mechanical components were tested and are at present preliminarly aligned in the experimental hall of ELETTRA.

\section{Conclusions}

We have presented the new circular polarization beam line presently under commissioning at ELETTRA.

The beam line is based on the use of two different monochromators with different angles of incidence. The use of concave holographically corrected gratings in the normal incidence monochromator and of a variable angle premirror in the grazing incidence one, strongly reduces the contribution of defocusing aberration.

The described circular polarization beam line appears to be a powerful beam line to exploit the radiation emitted from the ELETTRA electromagnetic elliptical wiggler.

\section{Acknowledgments}

It is a pleasure to thank two friends and a collegue for their help. I wish to record my gratitude to Prof. M. Piacentini and Dr. N. Zema for their useful advice and assistance along every stages of this project. I am also indebted to Dr. A. Derossi. 


\section{References}

[1] C.T. Chen, F. Sette, Y. Ma, S. Modesti, Phys. Rev. B 42, 7262 (1990).

[2] G. Schütz, W. Wagner, W. Wilhelm, P. Kienle, R. Zeller, R. Frahm, G. Materlik, Phys. Rev. Lett. 58, 737 (1987).

[3] T. Koide, T. Shidara, H. Fukutani, K. Yamaguchi, A. Fujimori, S. Kimura, Phys. Rev. B 44, 4697 (1991).

[4] C.T. Chen, Y.U. Idzerda, H.-J. Lin, G. Meigs, A. Chaiken, G.A. Prinz, G.H. Ho, Phys. Rev. B 48, 642 (1993).

[5] C.-C. Kao, C.T. Chen, E.D. Johnson, J.B. Hastings, H.-J. Lin, G.H. Ho, G. Meigs, J.-M. Brot, S.L. Hulbert, Y.U. Idzerda, C. Vettier, Phys. Rev. B 50, 9599 (1994).

[6] R.P. Walker, private communication; R.P. Walker, B. Diviacco, Rev. Sci. Instrum. 63, 332 (1992).

[7] H.A. Padmore, Rev. Sci. Instrum. 60, 1608 (1989).

[8] M. Neviere, P. Vincent, R. Petit, Nouv. Rev. Opt. 5, 65 (1974). 